\title{
Energy relaxation dynamics in vertically coupled multi-stacked InAs/GaAs quantum dots
}

\author{
Antaryami Mohanta, ${ }^{1, a)}$ Der-Jun Jang, ${ }^{1, b)}$ Shu-Kai Lu, ${ }^{1}$ Dah-Chin Ling, ${ }^{2}$ and J. S. Wang ${ }^{3}$ \\ ${ }^{1}$ Department of Physics, National Sun Yat-sen University, Kaohsiung 80424, Taiwan \\ ${ }^{2}$ Department of Physics, Tamkang University, Tamsui Dist., New Taipei City 25137, Taiwan \\ ${ }^{3}$ Department of Physics and Center for Nano-Technology, Chung-Yuan Christian University, \\ Chung-Li, 32023, Taiwan
}

(Received 5 October 2016; accepted 5 January 2017; published online 19 January 2017)

\begin{abstract}
Effect of GaAs spacer layer thickness $\left(d_{\mathrm{GaAs}}\right)$ on carrier capture, and the relaxation process is studied in multi-stacked InAs/GaAs quantum dots by photoluminescence and time-resolved photoluminescence. Auger scattering is the dominating process for carrier relaxation above $d_{\mathrm{GaAs}}$ of $15 \mathrm{~nm}$. At $d_{\mathrm{GaAs}}$ of $10 \mathrm{~nm}$, the carrier relaxation process is faster due to the combined effect of both single longitudinal optical phonon and Auger scattering resulting in higher photoluminescence intensity. The photoluminescence rise time corresponding to carrier capture and relaxation in quantum dots is longer at $3.06 \mathrm{eV}$ excitation than that at $1.53 \mathrm{eV}$ due to the effect of intervalley scattering in GaAs. Published by AIP Publishing. [http://dx.doi.org/10.1063/1.4974221]
\end{abstract}

Quantum dots (QDs) have been the potential candidates for different electronic and optoelectronic devices such as lasers, ${ }^{1}$ light emitting diodes, ${ }^{2}$ infrared detectors, ${ }^{3}$ single electron transistors, ${ }^{4}$ etc. Recently, InAs/GaAs based QDs self-assembled in the Stranski-Krastanov (SK) growth mode have been viewed with significant interest. ${ }^{1,5}$ Devices based on such QDs operate at wavelengths as long as $1.2 \mu \mathrm{m}$, and there is a great interest in extending this wavelength to $1.55 \mu \mathrm{m}$ and longer for use in fiber optic telecommunication systems. ${ }^{5}$ Multi-stacked (MS) InAs QDs with GaAs spacer have been the very promising device structures for the enhancement of the modal gain and the radiative efficiency. ${ }^{6,7}$ The GaAs spacer layer thickness $\left(d_{\mathrm{GaAs}}\right)$ has been reported to have a significant influence on the structural and optical properties of MS InAs/GaAs QDs. ${ }^{8-11}$ At larger $d_{\mathrm{GaAs}}$, QDs are not vertically correlated, and each QD layer exhibits properties similar to that of a single layer. However, properties of MS QDs are different from the single QDs layer for smaller $d_{\mathrm{GaAs}}$ due to the vertical correlation of QDs. ${ }^{9}$ Energy relaxation process in single layer selfassembled InAs/GaAs QDs have widely been reported. ${ }^{12-15}$ The intra-dot relaxation process has been expected to be slower than the radiative recombination process (several nanoseconds) due to the atomic-like discrete eigen states of QDs with energy spacing much larger than the longitudinal optical (LO) phonon energy, which is known as the "phonon bottleneck" effect. ${ }^{16}$ Experimental studies have revealed a fast intra-dot carrier relaxation $(<80 \mathrm{ps})$ which is in contrast to the phonon bottleneck effect. ${ }^{12-14}$ Recently, Kim et al. ${ }^{17}$ have observed the dominance of excited state emission in the photoluminescence (PL) spectra of MS InAs/GaAsSb QDs at and above the spacer layer thickness of $10 \mathrm{~nm}$. They have also reported that the increase in the PL intensity ratio of

\footnotetext{
${ }^{\text {a) }}$ Present address: EMPA-Swiss Federal Laboratories for Materials Science and Technology, Laboratory for Advanced Materials Processing, Feuerwerkerstrasse 39, 3602 Thun, Switzerland.

b) Author to whom correspondence should be addressed. Electronic mail: djjang@mail.nsysu.edu.tw.
}

excited state emission to ground state emission with increasing spacer layer thickness is attributed to the slower carrier relaxation rate which is in accordance with the phonon bottleneck effect. However, systematic investigation on the carrier relaxation process at different spacer layer thicknesses in InAs based MS QDs has not been reported which is inevitable to make them suitable for various electronic and optoelectronics applications.

In this letter, we have discussed the PL and timeresolved photoluminescence (TRPL) properties of MS InAs/ GaAs QDs at different $d_{\mathrm{GaAs}}$. PL peak energy is found to be increased monotonically with decreasing $d_{\mathrm{GaAs}}$ which is in contrast to the earlier reports. ${ }^{8-11,17} \mathrm{~A}$ standard TRPL upconversion apparatus is used to perform an extensive study on the carrier dynamics and the intervalley scattering in multistacked InAs/GaAs QDs. Single LO phonon scattering along with Auger scattering process is found to be responsible for the faster carrier relaxation at reduced $d_{\mathrm{GaAs}}(10 \mathrm{~nm})$.

The self-assembled MS InAs/GaAs QDs were grown by molecular beam epitaxy in the SK growth mode on (100)oriented n-type GaAs substrate. A buffer layer of $0.5 \mu \mathrm{m}$ thick GaAs, and $30 \mathrm{~nm}$ thick AlAs and $60 \mathrm{~nm}$ thick GaAs barrier layers were grown before 30 cycles of 2.6 monolayers of InAs with GaAs spacer layers were deposited. In order to cover the surface, $60 \mathrm{~nm}$ thick GaAs and $30 \mathrm{~nm}$ thick AlAs barrier layers, and then $5 \mathrm{~nm}$ GaAs cap layers were deposited.

MS InAs/GaAs QDs were characterized by transmission electron microscopy (TEM), atomic force microscopy (AFM), PL, and TRPL. AFM analysis reveals that the surface density of QDs in the investigated samples is about $5 \times 10^{10} \mathrm{~cm}^{-2}$. The vertical height of the QDs is about 6-7 nm and the width of $20-30 \mathrm{~nm}$. The PL measurements were performed at $35 \mathrm{~K}$ using a He closed-cycle cryostat. Frequency doubled (3.06 $\mathrm{eV}$ ) output of a mode-locked Ti: Sapphire laser (pulse width $\sim 100 \mathrm{fs}$, repetition rate $=80 \mathrm{MHz}$ ) were used as the excitation source. TRPL was measured at different photon energies $\left(E_{\mathrm{p}}\right)$ of the PL band at $35 \mathrm{~K}$. The experimental configuration is similar to that in the previous reports. ${ }^{18,19}$ 

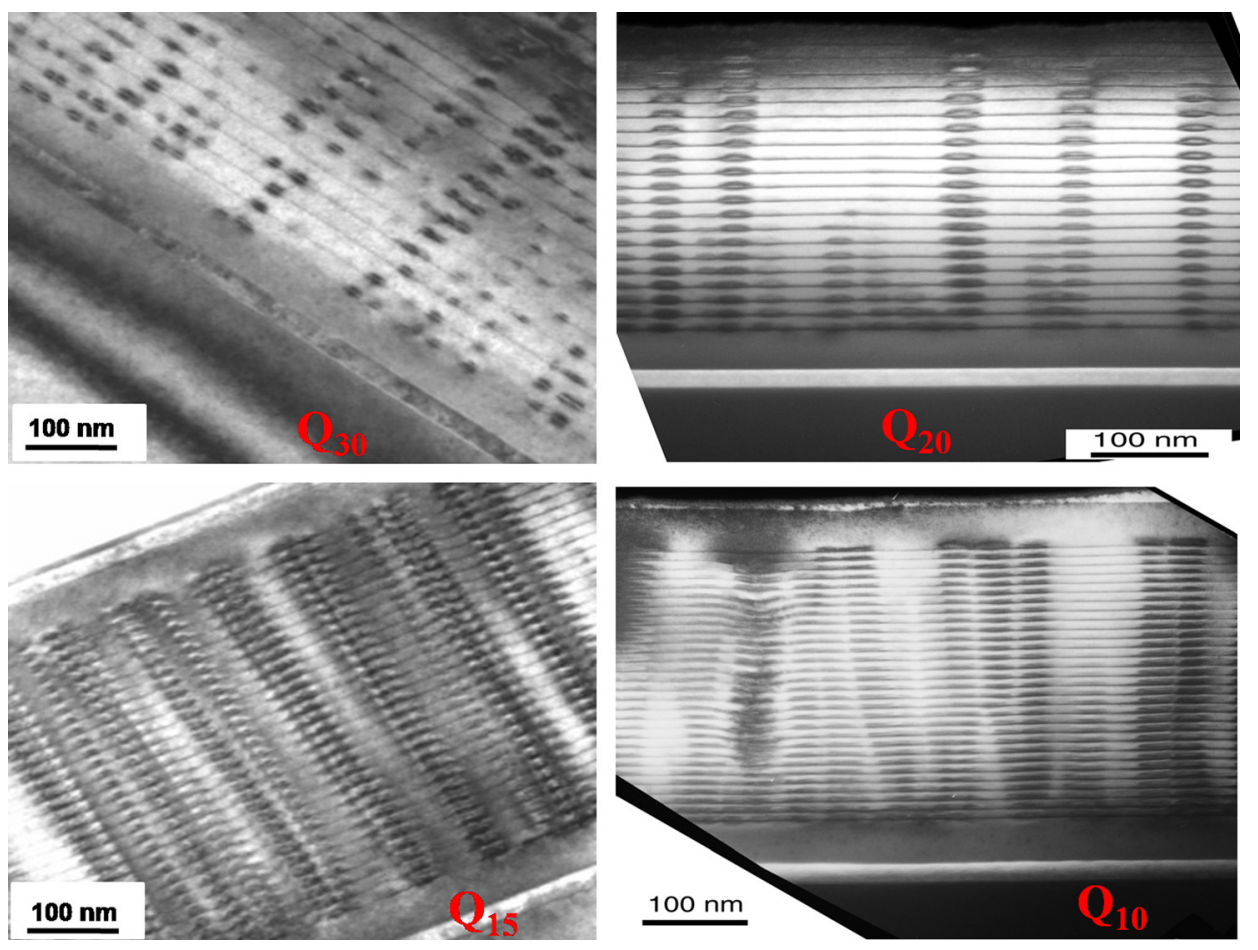

FIG. 1. Cross-sectional TEM images of the MS InAs/GaAs QDs having $d_{\mathrm{GaAs}}$ of $30,20,15$, and $10 \mathrm{~nm}$, respectively.
Figure 1 shows the cross-sectional TEM images of MS InAs/GaAs QDs with $d_{\mathrm{GaAs}}$ of $30,20,15$, and $10 \mathrm{~nm}$ which are assigned as $\mathrm{Q}_{30}, \mathrm{Q}_{20}, \mathrm{Q}_{15}$, and $\mathrm{Q}_{10}$, respectively. In $\mathrm{Q}_{30}$, QDs are formed in each layer; but there is no clear positional correlation. At reduced $d_{\mathrm{GaAs}}(<20 \mathrm{~nm})$, QDs are stacked and correlated vertically with each other. The vertical correlation of the QDs observed for $d_{\mathrm{GaAs}}<20 \mathrm{~nm}$ is caused by the strain field of the buried QDs in the underlying layer. The strain that resulted from the underlying layer usually causes atoms to migrate to the top regions of the buried QDs to form QDs in the upper layer. ${ }^{20}$ It has been reported that although the strain field from a single QD layer penetrates the GaAs spacer layer, it decays rapidly with the distance. In addition, the degree of strain field increases with an increase in the number of layers if $d_{\mathrm{GaAs}}$ is smaller than the decay length. ${ }^{9}$ Therefore, a complete vertical stacking of QDs is observed as the $d_{\mathrm{GaAs}}$ is reduced.

Figure 2(a) shows the PL spectra at $35 \mathrm{~K}$ of $\mathrm{Q}_{30}, \mathrm{Q}_{20}$, $\mathrm{Q}_{15}$, and $\mathrm{Q}_{10}$ at an excitation power $\left(P_{\text {exc }}\right)$ of $100 \mathrm{~mW}$ and an excitation energy $\left(E_{\text {exc }}\right)$ of $3.06 \mathrm{eV}$. All the PL spectra are comprised of two emission bands. The lower energy side emission is attributed to the ground state transition (GS) and the higher energy side to the emission from the first excited
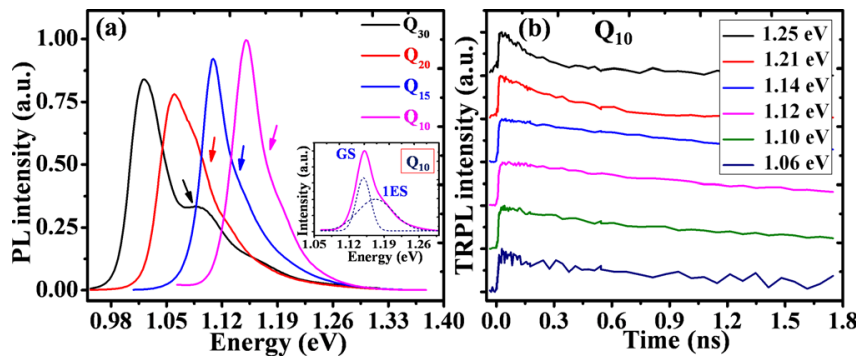

FIG. 2. (a) PL spectra at $35 \mathrm{~K}$ of $\mathrm{Q}_{30}, \mathrm{Q}_{20}, \mathrm{Q}_{15}$, and $\mathrm{Q}_{10}$ at $P_{\text {exc }}$ of $100 \mathrm{~mW}$ and $E_{\text {exc }}$ of $3.06 \mathrm{eV}$. The arrows indicate $1 \mathrm{ES}$. Inset shows the PL spectrum of $\mathrm{Q}_{10}$ with a multi-curve fitting to GS and 1ES. (b) The $E_{\mathrm{p}}$ dependent TRPL spectra at $35 \mathrm{~K}$ of $\mathrm{Q}_{10}$. state (1ES) marked by arrows in Fig. 2(a). Analysis of $P_{\text {exc }}$ dependent PL spectra (not shown) confirms that 1ES is the emission from the 1st excited state. The PL spectra are found to be dominated by GS for all $d_{\mathrm{GaAs}}$ which is in contrast to the earlier report by Kim et al. ${ }^{17}$ The peak energies of both GS and $1 \mathrm{ES}$ are determined using multi-curve fitting as shown in the inset of Fig. 2(a). The GS peak energy shows a prominent blue-shift of about $129 \mathrm{meV}$ as $d_{\mathrm{GaAs}}$ decreases from 30 to $10 \mathrm{~nm}$, i.e., from $\mathrm{Q}_{30}$ to $\mathrm{Q}_{10}$ which is in contrast to the previous reports that show a redshift due to the vertical interaction between the electronic states of the MS QDs. ${ }^{8-11} \mathrm{Kim}$ et al. ${ }^{17}$ have reported the nonmonotonic change of PL peak energy in the MS QDs with a decrease in spacer layer thickness due to the competing effects of increased compressive strain and vertical electronic coupling of the stacked QD layers. The observed blue-shift in Fig. 2(a) with decreasing $d_{\mathrm{GaAs}}$ has therefore been attributed to the dominating effect of compressive strain-driven intermixing of $\mathrm{In} / \mathrm{Ga}$ atoms at the interfaces between InAs QDs and GaAs spacer layers. ${ }^{17,21}$ Energy spacing $(\triangle \mathrm{E})$ between GS and 1ES decreases monotonically from 62 to $24 \mathrm{meV}$ as the $d_{\mathrm{GaAs}}$ decreases from 30 to $10 \mathrm{~nm}$ as shown in inset (a) of Fig. 3. The reduction in $\Delta \mathrm{E}$ with decreasing $d_{\mathrm{GaAs}}$ has been attributed to the compressive strain-driven enhanced interdiffusion of $\mathrm{In} / \mathrm{Ga}$ atoms at the interfaces between InAs QDs and GaAs spacer layers which is consistent with the observed blue-shift of GS peak energy with decreasing $d_{\text {GaAs }}$ [Fig. 2(a)]. ${ }^{17,21}$ The PL intensity initially decreases with a decrease in $d_{\mathrm{GaAs}}$, i.e., from $\mathrm{Q}_{30}$ to $\mathrm{Q}_{20}$, and then increases on further decrease in $d_{\mathrm{GaAs}}$, i.e., from $\mathrm{Q}_{20}$ to $\mathrm{Q}_{10}$. The initial decrease in PL intensity from $\mathrm{Q}_{30}$ to $\mathrm{Q}_{20}$ could be attributed to the disorder induced by strain-driven intermixing of the In/Ga atoms at the interfaces between InAs QDs and GaAs spacer layers. The increase in PL intensity from $\mathrm{Q}_{20}$ to $\mathrm{Q}_{10}$ could be associated with the dominating effect of faster carrier relaxation process due to smaller $\Delta \mathrm{E}$ at reduced $d_{\mathrm{GaAs}}$. 


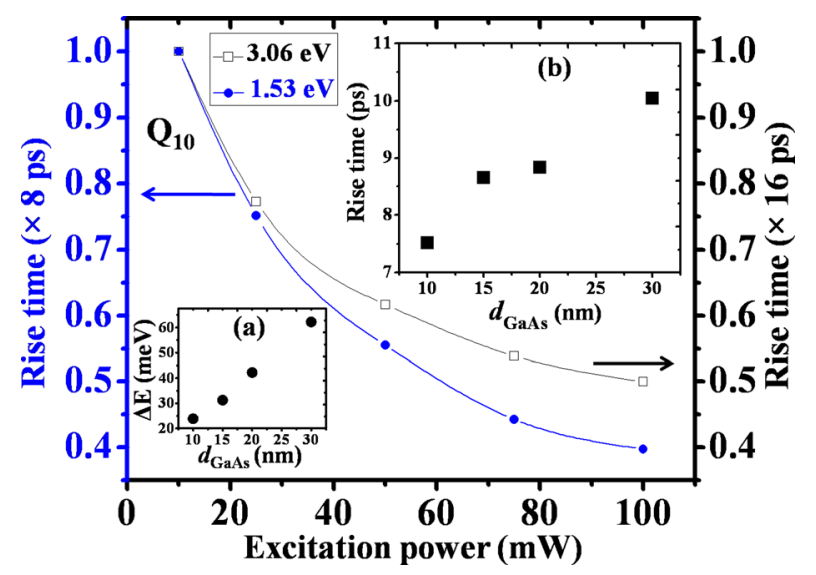

FIG. 3. Variation of rise time at GS energy with $P_{\text {exc }}$ at $E_{\text {exc }}$ of 1.53 and $3.06 \mathrm{eV}$ for $\mathrm{Q}_{10}$ as representative of all QDs. The insets (a) and (b) show a variation of $\Delta \mathrm{E}$ and a rise time at GS energy, respectively, with $d_{\mathrm{GaAs}}$ at $P_{\text {exc }}$ of $100 \mathrm{~mW}$ and $E_{\mathrm{exc}}$ of $3.06 \mathrm{eV}$.

In order to understand the carrier relaxation process, the TRPL spectra at $35 \mathrm{~K}$ were recorded at different $P_{\text {exc }}$ and $E_{\mathrm{p}}$ at fixed $E_{\text {exc }}$ of 1.53 and $3.06 \mathrm{eV}$. Figure 2(b) shows the TRPL spectra at $35 \mathrm{~K}$ of $\mathrm{Q}_{10}$ at different $E_{\mathrm{p}}$, and at fixed $E_{\text {exc }}$ of $3.06 \mathrm{eV}$ and $P_{\mathrm{exc}}$ of $100 \mathrm{~mW}$ as representative of all the samples and excitation conditions. Exponential function was used to determine the rise time by fitting the TRPL spectra. The rise time has been attributed to the carrier capture from the GaAs layers and intra-dot relaxation time within the QDs, and is shown in Fig. 4 at different $E_{\mathrm{p}}$ at fixed $E_{\text {exc }}$ of $3.06 \mathrm{eV}$ and $P_{\text {exc }}$ of $100 \mathrm{~mW}$. The $E_{\mathrm{p}}$ dependence of rise time represents both the GS and 1ES emission band which can be envisaged from its doublet behavior in Fig. 4. In $\mathrm{Q}_{30}$, the rise time of GS emission energy is longer than that of 1ES. This indicates that the carrier capture rate of $1 E S$ energy level is faster than that of GS. In other words, 1ES energy level is first filled by the carrier capture and then the carriers are relaxed down towards the GS energy level to occupy through carrier relaxation process. The 1ES energy level therefore acts as intermediate state in the relaxation process in $\mathrm{Q}_{30}$ which is in contrast to the earlier report by Bogaart et al. ${ }^{15}$ They reported that the rise time of the first excited state is longer than that of the ground state in InAs quantum dots, and attributed to the relaxation of carriers through the continuum background, followed by a single LO-phonon emission towards the QD ground state according to the continuum relaxation model. Carrier relaxation via the polaron decay is about 4.5-7 times slower than the observed maximum rise time $(\sim 10 \mathrm{ps})$ in $\mathrm{Q}_{30} \cdot{ }^{22}$ The $\Delta \mathrm{E}$ in $\mathrm{Q}_{30}$ is about $62 \mathrm{meV}$. Thus, the carrier relaxation by a single LO phonon emission is not possible as the LO phonon energy in InAs is $\sim 29 \mathrm{meV}$. Multi-phonon scattering processes ${ }^{23}$ and Auger-scattering ${ }^{13,14,24}$ have been proposed to be responsible for the rapid relaxation of carriers in a QD on the time scales of picoseconds. Multi-phonon scattering process is still a too slow process ( $>10 \mathrm{ps}$ ) and is effective at low excitation level as Auger-like carrier-carrier (cc) scattering process dominates at a high excitation level. ${ }^{13,14}$ The dot density is about $5 \times 10^{10} \mathrm{~cm}^{-2}$, and the carrier density at $100 \mathrm{~mW}$ in the first GaAs layer is $\sim 10^{13} \mathrm{~cm}^{-2}$ which is sufficient for the Auger scattering to dominate the carrier relaxation process in $\mathrm{Q}_{30}$. The decrease in PL rise time with increasing $P_{\text {exc }}$ is the characteristic feature of the Auger process ${ }^{13,14}$ that can be envisaged from Fig. 3 which further supports the dominance of Auger scattering in carrier relaxation process. Similar behavior is observed in $\mathrm{Q}_{20}$ and $\mathrm{Q}_{15}$. However, the rise time of both GS and $1 E S$ in $\mathrm{Q}_{10}$ is approximately the same which indicates that the filling rate of carriers in both GS and 1ES energy levels is same which is in contrast to $\mathrm{Q}_{30}, \mathrm{Q}_{20}$, and $\mathrm{Q}_{15}$. The $\Delta \mathrm{E}$ in $\mathrm{Q}_{10}$ is $24 \mathrm{meV}$ which is comparable to the LO phonon energy $(29 \mathrm{meV})$ in InAs. Therefore, the carrier relaxation by a single LO phonon emission is possible. The variation of rise time of the GS energy in $\mathrm{Q}_{10}$ with $P_{\text {exc }}$ is shown in Fig. 3. The rise time is found to be decreased with

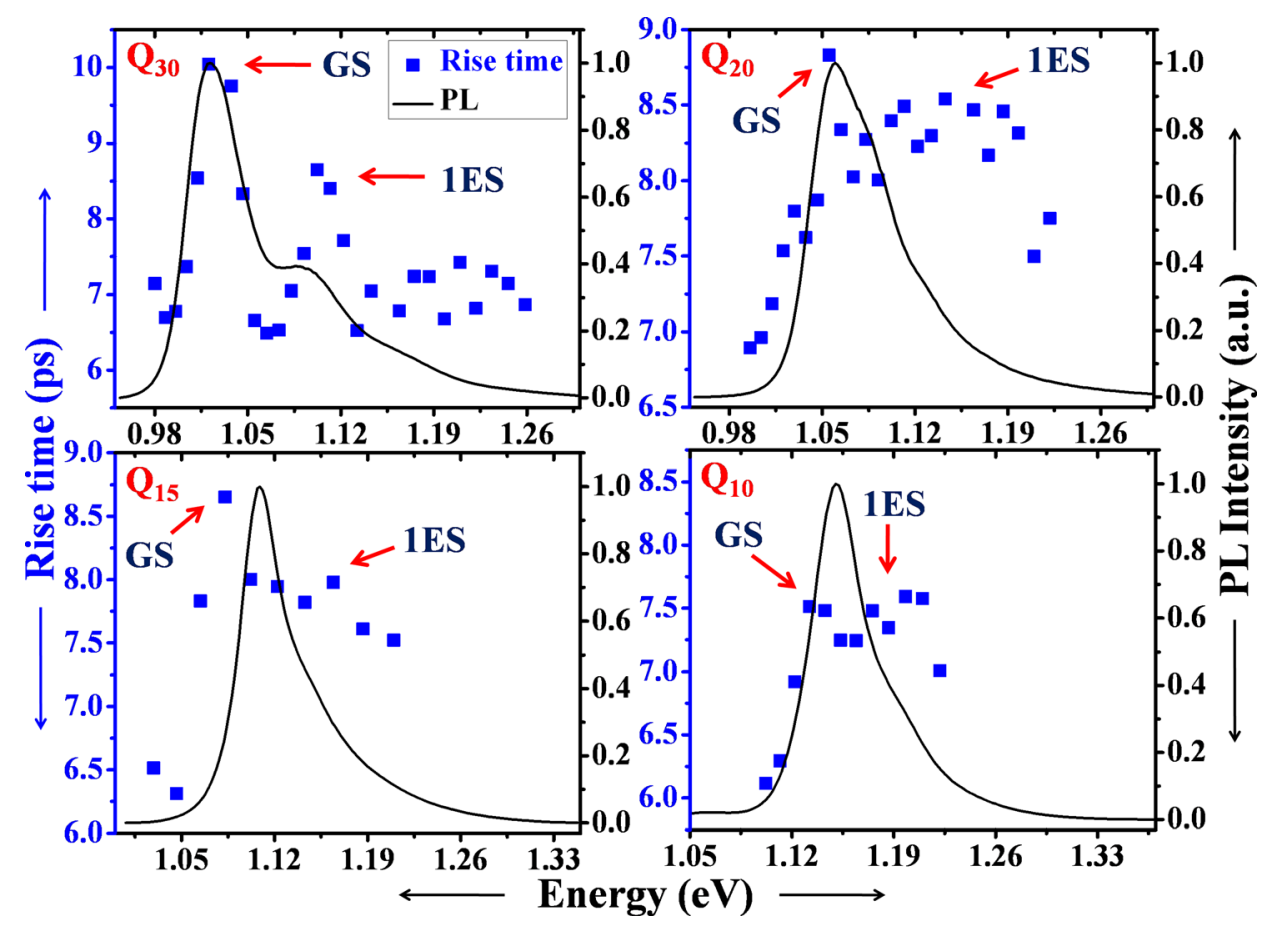

FIG. 4. Rise time of $\mathrm{Q}_{30}, \mathrm{Q}_{20}, \mathrm{Q}_{15}$, and $\mathrm{Q}_{10}$ at different $E_{\mathrm{p}}$ at $E_{\mathrm{exc}}$ of $3.06 \mathrm{eV}$ (घ). The solid curve represents the corresponding PL spectra. 
an increase in $P_{\text {exc }}$ which indicates that Auger scattering is also responsible for the carrier relaxation process in $\mathrm{Q}_{10}$ at $P_{\text {exc }}=100 \mathrm{~mW}$ in addition to carrier relaxation by single LO phonon emission. The occurrence of carrier relaxation in $\mathrm{Q}_{10}$ both by single phonon emission and Auger scattering at 100 $\mathrm{mW}$ explains the same filling rate of carriers in GS and 1ES energy levels. Furthermore, the rise time of GS emission energy is found to be decreased with a decrease in $d_{\mathrm{GaAs}}$ [inset (b) of Fig. 3]. The decrease in $\Delta \mathrm{E}$ with a decrease in $d_{\mathrm{GaAs}}$ involves less number of phonons for scattering and facilitates the carrier relaxation process by Auger scattering which results in a decrease in rise time with a decrease in $d_{\mathrm{GaAs}}$.

The rise time in $\mathrm{Q}_{10}$ is found to be more at $E_{\text {exc }}$ of $3.06 \mathrm{eV}$ than that at $1.53 \mathrm{eV}$ which is obvious from Fig. 3 . Similar behavior of rise time with $E_{\text {exc }}$ is also observed in $\mathrm{Q}_{30}, \mathrm{Q}_{20}$, and $\mathrm{Q}_{15}$. This behavior is not associated with the difference in photogenerated carrier density $\left(\sim 1 \times 10^{13} \mathrm{~cm}^{-2}\right.$ for $1.53 \mathrm{eV}$ and $4 \times 10^{13}$ for $3.06 \mathrm{eV}$ in the first GaAs layer) since the rise time decreases with an increase in $P_{\text {exc }}$ (Fig. 3). As both $E_{\text {exc }}$ generates the carriers in the GaAs spacer layer, it is important to consider the band structure of GaAs. The band gap of GaAs is about $1.519 \mathrm{eV}$, and the bottom of the conduction band is described by the $\Gamma$-valley. The L-valley and $\mathrm{X}$ valley are located in the conduction band at about 1.815 and $1.981 \mathrm{eV}$ above the valence band maxima. For $1.53 \mathrm{eV}$ excitation, the carriers are generated in the $\Gamma$-valley and then relax down to the bottom of the conduction band of GaAs within the $\Gamma$ - valley. Subsequently, carriers are captured by the InAs QDs from the GaAs spacer layer. However, for $3.06 \mathrm{eV}$ excitation, the carriers have enough excess energy such that the carriers can be scattered from the $\Gamma$-valley to L-valley and Xvalley, and return back to $\Gamma$-valley by the intervalley scattering process. The difference in rise times at $E_{\text {exc }}$ of 1.53 and $3.06 \mathrm{eV}$ varies from $\sim 8$ to $4.5 \mathrm{ps}$ as the $P_{\mathrm{exc}}$ increases. However, the intervalley scattering time in $\mathrm{GaAs}$ from $\Gamma$ - to L-valley is reported to be within $100 \mathrm{fs}$ and that for the L- to $\Gamma$-valley is about 2 ps. $^{25}$ Thus, the observed higher value ( 4.5 $-8 \mathrm{ps}$ ) of difference in rise times at $3.06 \mathrm{eV}$ and $1.53 \mathrm{eV}$ indicates an effective intervalley scattering between $\Gamma$ - and Xvalley, and a slower carrier capture and relaxation at $3.06 \mathrm{eV}$ due to more excess energy. Moreover, the rise time at $1.53 \mathrm{eV}$ decreases at a faster rate than that at $3.06 \mathrm{eV}$ with increasing $P_{\text {exc }}$ as shown in Fig. 3 which indicates that Auger scattering is more effective as the $E_{\text {exc }}$ is reduced due to less excess energy.

In summary, we have shown the effect of $d_{\mathrm{GaAs}}$ on both $\mathrm{PL}$ and carrier relaxation process in MS InAs/GaAs QDs. With a decrease in $d_{\mathrm{GaAs}}$, the $\Delta \mathrm{E}$ decreases which becomes comparable to the LO phonon energy $(\sim 29 \mathrm{meV})$ at $d_{\mathrm{GaAs}}=10 \mathrm{~nm}$ in $\mathrm{Q}_{10}$. Auger scattering is found to be responsible for the carrier relaxation process in the investigated QDs. The carrier relaxation process in $\mathrm{Q}_{10}$ is faster in comparison to that in QDs of larger $d_{\mathrm{GaAs}}(\geq 15 \mathrm{~nm})$ due to the effect of both single LO phonon and Auger scattering. The rise time, i.e., the time for carrier capture and intra-dot relaxation is found to be longer at $E_{\mathrm{exc}}=3.06 \mathrm{eV}$ than that at $1.53 \mathrm{eV}$ due to intervalley scattering process. The lowest carrier capture and intra-dot relaxation time is observed to be $\sim 3 \mathrm{ps}$ in $\mathrm{Q}_{10}$ at $P_{\mathrm{exc}}$ of $100 \mathrm{~mW}$ and $E_{\mathrm{exc}}$ of $1.53 \mathrm{eV}$ which is much smaller than the value reported earlier in case of single layer QDs. ${ }^{12,13}$ Therefore, multi-stacked InAs/GaAs QDs with complete vertical alignment at reduced $d_{\mathrm{GaAs}}$ can be employed for optoelectronic device applications due to the dominating effect of faster intra-dot carrier relaxation process and a higher modal gain. ${ }^{6,7}$

This research was supported by the Ministry of Science and Technology of the Republic of China, Grant No. 1042112-M-110-010.

${ }^{1}$ H.-Y. Liu, B. Xu, Y.-Q. Wei, D. Ding, J.-J. Qian, Q. Han, J.-B. Liang, and Z.-G. Wang, Appl. Phys. Lett. 79, 2868 (2001).

${ }^{2}$ V. Tasco, M. T. Todaro, M. De Vittorio, M. De Giorgi, R. Cingolani, A. Passaseo, J. Ratajczak, and J. W. Katcki, Appl. Phys. Lett. 84, 4155 (2004).

${ }^{3}$ H. C. Liu, M. Gao, J. McCaffrey, Z. R. Wasilewski, and S. Fafard, Appl. Phys. Lett. 78, 79 (2001).

${ }^{4}$ H. Drexler, D. Leonard, W. Hansen, J. P. Kotthaus, and P. M. Petroff, Phys. Rev. Lett. 73, 2252 (1994).

${ }^{5}$ C. N. Allen, P. J. Poole, P. Marshall, J. Fraser, S. Raymond, and S. Fafard, Appl. Phys. Lett. 80, 3629 (2002).

${ }^{6}$ C. H. Roh, Y. J. Park, K. M. Kim, Y. M. Park, E. K. Kim, and K. B. Shim, J. Cryst. Growth 226, 1 (2001).

${ }^{7}$ S. Rouvimov, Z. Liliental-Weber, W. Swider, J. Washburn, E. R. Weber, A. Sasaki, A. Wakahara, Y. Furkawa, T. Abe, and S. Noda, J. Electron. Mater. 27, 427 (1998).

${ }^{8}$ G. S. Solomon, J. A. Trezza, A. F. Marshall, and J. S. Harris, Jr., Phys. Rev. Lett. 76, 952 (1996).

${ }^{9}$ M. A. Migliorato, L. R. Wilson, D. J. Mowbray, M. S. Skolnick, M. AlKhafaji, A. G. Cullis, and M. Hopkinson, J. Appl. Phys. 90, 6374 (2001).

${ }^{10}$ M. S. Miller, J.-O. Malm, M.-E. Pistol, S. Jeppesen, B. Kowalski, K. Georgsson, and L. Samuelson, J. Appl. Phys. 80, 3360 (1996).

${ }^{11}$ J. He, Y. C. Zhang, B. Xu, and Z. G. Wang, J. Appl. Phys. 93, 8898 (2003).

${ }^{12}$ R. Heitz, M. Veit, N. N. Ledentsov, A. Hoffmann, D. Bimberg, V. M. Ustinov, P. S. Kop'ev, and Zh. I. Alferov, Phys. Rev. B 56, 10435 (1997).

${ }^{13}$ D. Morris, N. Perret, and S. Fafard, Appl. Phys. Lett. 75, 3593 (1999).

${ }^{14}$ B. Ohnesorge, M. Albrecht, J. Oshinowo, A. Forchel, and Y. Arakawa, Phys. Rev. B 54, 11532 (1996).

${ }^{15}$ E. W. Bogaart, J. E. M. Haverkort, T. Mano, T. van Lippen, R. Nötzel, and J. H. Wolter, Phys. Rev. B 72, 195301 (2005).

${ }^{16}$ R. Heitz, H. Born, F. Guffarth, O. Stier, A. Schliwa, A. Hoffmann, and D. Bimberg, Phys. Rev. B 64, 241305(R) (2001).

${ }^{17}$ Y. Kim, K.-Y. Ban, A. Boley, D. J. Smith, and C. B. Honsberg, Appl. Phys. Lett. 107, 173109 (2015).

${ }^{18}$ D.-J. Jang, W.-C. Chou, C.-S. Yang, C.-T. Kuo, and M.-E. Lee, Jpn. J. Appl. Phys., Part 1 42, 7370 (2003).

${ }^{19}$ A. Mohanta, D.-J. Jang, G.-T. Lin, Y.-T. Lin, and L. W. Tu, J. Appl. Phys. 110, 023703 (2011).

${ }^{20}$ B. Legrand, J. P. Nys, B. Grandidier, D. Stiévenard, A. Lemaître, J. M. Gérard, and V. Thierry-Mieg, Appl. Phys. Lett. 74, 2608 (1999).

${ }^{21}$ X. C. Wang, S. J. Xu, S. J. Chua, Z. H. Zhang, W. J. Fan, C. H. Wang, J. Jiang, and X. G. Xie, J. Appl. Phys. 86, 2687 (1999).

${ }^{22}$ S. Sauvage, P. Boucaud, F. Bras, G. Fishman, R. P. S. M. Lobo, F. Glotin, R. Prazeres, J.-M. Ortega, and J.-M. Gérard, Phys. Status Solidi (b) 238, 254 (2003).

${ }^{23}$ T. Inoshita and H. Sakaki, Phys. Rev. B 46, 7260 (1992).

${ }^{24}$ U. Bockelmann and T. Egeler, Phys. Rev. B 46, 15574 (1992).

${ }^{25}$ J. Shah, B. Deveaud, T. C. Damen, W. T. Tsang, A. C. Gossard, and P. Lugli, Phys. Rev. Lett. 59, 2222 (1987). 